\title{
ANÁLISIS DE LAS TEORÍAS DE MOTIVACIÓN DE CONTENIDO: UNA APLICACIÓN AL MERCADO LABORAL DE CHILE DEL AÑO 2009
}

\section{ANALYSIS OF THE MOTIVATION THEORIES OF CONTENT: AN APPLICATION TO CHILE'S LABOR MARKET OF 2009}

\author{
Luis Araya-Castillo* \\ Margarita Pedreros-Gajardo**
}

\begin{abstract}
RESUMEN
Actualmente es importante estudiar las teorías de motivación de contenido, por cuanto se enfocan en las necesidades que motivan a los trabajadores. Por esta razón, la presente investigación hizo uso del análisis factorial de componentes principales con el objetivo de configurar las principales teorías de contenido, utilizando como muestra a trabajadores chilenos. Los resultados validan los supuestos de la investigación, por cuanto se demuestra que estas teorías están relacionadas, pero que al mismo tiempo difieren en la importancia que atribuyen a las necesidades. En este escenario, se concluye que las diferentes teorías de motivación de contenido comparten un conjunto de premisas acerca de las necesidades humanas, aun cuando las agrupaciones resultantes ( $y$ pesos relativos de las necesidades) sean diferentes.
\end{abstract}

PALABRAS CLAVE: CHILE * TEORÍA * MOTIVACIÓN * TRABAJADORES * ANÁLISIS ESTADÍSTICO

\section{ABSTRACT}

Currently, decision makers must understand the needs that motivate workers, because they focus on the needs that motivate to the workers. For this reason, the present study had used the factorial analysis of the principal components, in order to configure the main theories of content. The findings validate these assumptions showing that these theories are interrelated, but at the same time, these differ in terms of the level of importance attributable to the needs. In this context, it is concluded that different theories of content motivation share a set of assumptions about human needs, even of the cluster resulting (and relative weights of the needs) are different.

KEYWORDS:CHILE * THEORY * MOTIVATION * WORKERS * STATISTICAL ANALYSIS

\footnotetext{
* Departamento de Administración, Facultad de Economía y Negocios de la Universidad de Chile. laraya@unegocios.cl

** Facultad de Economía y Negocios de la Universidad de Chile. margarita.pedreros.g@gmail.com
} 


\section{INTRODUCCIÓN}

Actualmente, la gestión de recursos humanos juega un papel de importancia en la estrategia competitiva de las empresas. Es así que el recurso humano (o capital humano) es visto como un recurso estratégico, ya que altos niveles de desempeño en los trabajadores se traducen en altos niveles de rendimiento en las organizaciones.

En este contexto, los tomadores de decisiones no pueden asumir que los trabajadores realizarán sus labores por el solo hecho de existir un contrato de empleo, ya que sus intereses pueden ser distintos a los objetivos organizacionales. La teoría de agencia pone de manifiesto esta situación, al señalar que los individuos (trabajadores) tienen necesidades que en muchos casos son distintas a las de los administradores (o de los dueños del capital).

Lo anterior podría tener serias repercusiones en la obtención de ventajas competitivas; es decir, en la consecución de un nivel de rentabilidad superior al promedio de la industria. Por lo tanto, en busca de coordinar los esfuerzos de cada uno de los integrantes de la organización, los tomadores de decisiones deben ser capaces de diseñar planes adecuados de motivación.

Esto implica que los tomadores de decisiones deben identificar las necesidades de sus trabajadores $y$ ofrecerles los medios para que las puedan satisfacer. Es así como, trabajadores satisfechos se mostrarán motivados por su trabajo, lo cual se reflejará en mayores niveles de desempeño y compromiso organizacional. En último término, esto permite que la empresa pueda cumplir sus objetivos en forma eficaz y eficiente (es decir, que pueda obtener ventajas competitivas).

Dado esto, la presente investigación tiene como propósito configurar y analizar las principales teorías de motivación de contenido, en una muestra representativa de trabajadores chilenos. Con lo anterior se pretende aportar a la literatura, por cuanto se estudian y configuran las diferentes teorías de motivación de contenido a través de un mismo conjunto de preguntas (ítems).

Aun cuando los resultados expuestos son de carácter descriptivo, pueden ser utilizados como base en la realización de otros estudios (por ejemplo, que busquen relacionar los niveles de motivación con los de desempeño) o en la práctica profesional (por ejemplo, con el diseño de planes de motivación).

\section{REVISIÓN DE LA LITERATURA}

\subsection{MOTIVACIÓN}

La motivación se refiere a aquello que hace que la gente actúe o se comporte de determinadas maneras (Hampton, 2000). Es decir, la motivación corresponde a una serie de impulsos o deseos, los cuales fomentan cierto comportamiento (Flores, 1996). Por lo tanto, cuando se estudia la motivación se parte de la premisa de que incide notablemente en todas las acciones que las personas pueden realizar (Soler y Chirolde, 2010).

Sin embargo, cuando se habla de motivación, no solo se hace referencia a las personas, sino también al sujeto forjador de dicha motivación (Robbins y Coulter, 2005). Por lo cual, los tomadores de decisiones deben conocer las condiciones en las cuales a los trabajadores puede motivárseles para que efectúen su trabajo, al mismo tiempo que se cumplen los objetivos organizacionales.

Con este objetivo, el primer paso de los tomadores de decisiones debe consistir en reconocer que el ser humano sigue sus propios intereses, tal como los definen sus necesidades (Robbins y Coulter, 2005). Esto implica que los tomadores de decisiones deben reconocer que en una organización se necesita de los esfuerzos y colaboración de los trabajadores, ya que sin estos no es posible obtener posiciones competitivas en el mercado.

\subsection{NECESIDAD}

Las personas se mueven a través de motivos, es decir, en la búsqueda de satisfacer ciertas necesidades. Esto implica que una vez satisfecha la necesidad, se reduce el impulso que motivó la conducta (Ferrell y Hirt, 2003; Robbins y Coulter, 2005). Lo anterior tiene repercusiones en el estudio de las necesidades, por cuanto estas no son observables, sino son constructos que se derivan de sus efectos (Todt, 1991). 
Las necesidades humanas pueden abarcar un amplio espectro. Como forma de simplificación, estas se pueden dividir en dos grupos: necesidades básicas y necesidades secundarias. Las primeras, se relacionan con la existencia humana (sed, abrigo, vivienda, etc.), en tanto que las segundas, tienen relación con la satisfacción de necesidades de orden superior (sociales, ego, etc.). Las necesidades primarias son innatas, en tanto que las secundarias se aprenden. Esto implica que el ser humano puede vivir sin la satisfacción de las necesidades secundarias, pero la vida sería entonces menos placentera (Hampton, 2000).

En este contexto se argumenta que las necesidades pueden ser provocadas por procesos internos de un determinado tipo, pero más frecuentemente (cuando ya se encuentran dispuestas) son consecuencia de la aparición eficaz de una presión del entorno o de la anticipación imaginada de las mismas (Soriano, 2001).

\subsection{TEORÍAS MOTIVACIONALES}

Las teorías motivacionales se pueden dividir entre aquellas de contenidos y las de proceso (Naranjo, 2009). Las primeras estudian y consideran aspectos que pueden motivar a las personas, en tanto que las segundas estudian y tienen en cuenta el proceso de pensamiento por el cual estas se motivan.

Dentro de las teorías de motivación de contenido se pueden mencionar las de Maslow, Alderfer, McClelland, Herzberg y McGregor (Teoría X e Y). Sin embargo, en la presente investigación solo se analizan las de Maslow, Alderfer, McClelland y Herzberg, ya que se enfocan en determinar las necesidades humanas en función de tipologías predeterminadas. Esto no sucede con la Teoría X e Y, donde no se conoce a priori el número de dimensiones a obtener.

\subsubsection{TEORÍA DE MASLOW}

Maslow (1943) señala que las personas se encuentran motivadas por cinco tipos de necesidades: fisiológicas (alimento, agua y abrigo), de seguridad (protección, orden y estabilidad), sociales (afecto, amistad y sentido de pertenencia), autoestima (prestigio, status y autoestima) y autorrealización (autosatisfacción). Estas necesidades humanas están ordenadas según una jerarquía, donde unas son prioritarias y solo cuando estas están cubiertas, se puede ascender a necesidades de orden superior (Santrock, 2002).

Las necesidades se satisfacen en el siguiente orden, primero, las necesidades básicas (fisiológicas y de seguridad) y luego las necesidades secundarias (social, autoestima $y$ autorrealización). Esto es porque las personas buscan satisfacer sus necesidades básicas y luego ascender en la búsqueda de satisfacer necesidades más complejas (Daft, 2004).

Un aspecto importante en la Teoría de Maslow es que una vez satisfechas una serie de necesidades, estas dejan de fungir como motivadores (Robbins y Coulter, 2005). Esto implica que para motivar a los trabajadores en la realización de sus labores es necesario permitirles satisfacer sus necesidades insatisfechas.

A pesar de que existe escasa evidencia empírica que respalde la Teoría de Maslow, esta goza de amplia aceptación (Certo, 2001). La literatura no apoya la existencia de la jerarquía exactamente como fue concebida por el autor (Wahba y Bridwell, 1976). Asimismo, se señala que esta teoría puede oscurecer las diferencias de las necesidades secundarias que varían según las personas y las culturas (Daft, 2004). No obstante, existe evidencia empírica que apoya la división de las necesidades en primarias y secundarias. Además, se ha demostrado que las necesidades que motivan a las personas son aquellas que no están satisfechas.

\subsubsection{TEORÍA DE ALDERFER}

Algunos autores señalan que la tipología de necesidades propuesta por Aldefer no se basa en elementos nuevos, sino que se fundamenta en la Teoría de Maslow. Aun cuando esto es correcto, se puede argumentar que Alderfer (1969) realiza algunas críticas a la Teoría de Maslow (Naranjo, 2009), lo cual lleva a que su trabajo se diferencie en tres aspectos (Bonillo y Nieto, 2002).

En primer lugar, reduce las cinco necesidades de Maslow a solo tres. Estas necesidades son las de existencia (necesidad de bienestar 
físico), relación (relaciones interpersonales) y crecimiento (desarrollo y crecimiento personal). En segundo lugar, Alderfer (1969) argumenta que en la aparición de una nueva necesidad, no es imprescindible que se hayan cubierto (satisfechas) de manera suficiente las inferiores. Además, este mismo autor encontró que el movimiento de la jerarquía de necesidades no es solo en orden ascendente, por cuanto las personas pueden retroceder en la jerarquía con el objetivo de satisfacer una necesidad ya satisfecha.

A pesar de estas diferencias, se pueden encontrar ciertas similitudes entre ambas teorías de motivación. Es así como, la necesidad de existencia de Alderfer puede representar a las necesidades fisiológicas y de seguridad de Maslow. Algo similar ocurre con la necesidad de relación (la cual representa la necesidad social de Maslow) y de crecimiento (la cual representa las necesidades de autoestima y autorrealización de Maslow).

\subsubsection{TEORÍA DE MCCLELLAND}

McClelland (1961) señala que existen tres motivaciones en las personas, las cuales son las necesidades de logro, afiliación y poder. Algunos autores sostienen que estas necesidades son motivaciones sociales, ya que se aprenden de una manera no consciente, como producto de enfrentarse activamente al medio. Dado esto, su importancia radica en que predisponen a las personas a comportarse en formas que afectan de manera crítica el desempeño en muchos trabajos y tareas (Naranjo, 2009).

Debido a lo anterior, las personas con una alta necesidad de logro, buscan distinguirse por hacer bien las cosas $y$ disfrutan de situaciones en las que pueden tomar responsabilidades. Asimismo, las personas que tienen una alta necesidad de poder, buscan controlar a los demás y que se haga lo que ellas desean. Por su parte, las personas con una alta necesidad de afiliación tienden a interesarse $y$ a pensar con frecuencia acerca de la calidad de sus relaciones personales (Santrock, 2002).

Una comparación de la Teoría de McClelland con los estudios de Maslow, permite señalar que el poder podría considerarse una clase particular de necesidad social o de estimación, por referirse a las relaciones con las personas y el estatus; también puede guardar relación con la necesidad de seguridad. La afiliación es un sinónimo suavizado de lo que Maslow llama necesidad social; en tanto que el logro puede recordar algunos patrones conductuales relativos a la autoestima o la autorrealización (Daft, 2004).

\subsubsection{TEORÍA DE MOTIVACIÓN E HIGIENE}

La Teoría de Herzberg (Herzberg, Mausner y Snyderman, 1959) centra su atención en el trabajo en sí mismo como fuente principal de satisfacción. Esta teoría subraya la importancia de las características del puesto y las prácticas organizacionales (Robbins y Coulter, 2005).

Herzberg consideraba que el trabajo es la actividad más importante del individuo (Batista, Gálvez e Hinojosa, 2010). De acuerdo a esto, concluyó que la satisfacción laboral y la insatisfacción laboral son producto de dos tipos de experiencias distintas (modelo de dos factores). Es decir, Herzberg estableció que los factores que generan insatisfacción en los trabajadores son de naturaleza totalmente diferente a la de los factores que producen satisfacción (Brunstein, Romero y Albarrán, 2007).

Por un lado, los factores que generan satisfacción se conocen como intrínsecos, motivadores o satisfactores, $y$ por otro lado, los factores que producen insatisfacción se consideran como extrínsecos, de higiene, insatisfactores o de apoyo (Bonillo y Nieto, 2002). Los factores intrínsecos se relacionan con el contenido del trabajo (el trabajo en sí mismo, la responsabilidad y el reconocimiento, entre otros) y los factores extrínsecos lo hacen con el contexto laboral (relaciones con el supervisor, el salario y las relaciones con los compañeros, entre otros).

Dado esto, se puede argumentar que cuando las personas hablan de sentirse bien o satisfechas, hacen referencia a los factores intrínsecos, los cuales se relacionan con la satisfacción laboral cuando están presentes, pero no con la insatisfacción cuando están ausentes. Considerando esta situación, cuando los empleados hablan de sentirse insatisfechos con el trabajo se refieren a los factores externos del mismo (aunque relacionados), los 
cuales se asocian con la insatisfacción laboral cuando están ausentes, pero no con la satisfacción cuando están presentes.

\section{METODOLOGÍA}

Con el objetivo de determinar las dimensiones (necesidades) de las tipologías (teorías) de motivación estudiadas, se aplica el análisis factorial de componentes principales con Rotación Varimax (Hair et ál., 2005). Sin embargo, lo anterior no garantiza que los resultados obtenidos tengan significancia estadística, razón por cual a las dimensiones encontradas se les realizan los análisis de validez y confiabilidad correspondientes (Nunnally, 1978; Sánchez y Sarabia, 1999). Sumado a esto, se realiza el análisis de unidimensionalidad a las dimensiones propuestas, por cuanto se analiza si miden un único constructo (Hair et ál., 1998).

\subsection{DEFINICIÓN DE OBJETIVOS E HIPÓTESIS DE TRABAJO}

El análisis de las teorías de motivación expuestas, permite argumentar que comparten un conjunto de premisas en la configuración de los tipos de necesidades. Es decir, aun cuando estas teorías trabajan con supuestos teóricos diferentes, la agrupación que realizan de las necesidades presenta similitudes. Dado esto, en la presente investigación se formulan las siguientes hipótesis:

$\diamond \quad$ Hipótesis 1: las teorías de motivación de contenido (Maslow, Alderfer, McClelland $y$ Herzberg), se pueden configurar con un mismo conjunto de preguntas (o ítems).

$\diamond \quad$ Hipótesis 2: las teorías de motivación de contenido (Maslow, Alderfer, McClelland $y$ Herzberg), presentan diferencias en el peso relativo (importancia) que asignan a las necesidades.

Dado esto, el objetivo general de la presente investigación consiste en configurar las teorías de motivación de contenido a través de una muestra representativa de trabajadores chilenos. Esto permite el planteamiento de los siguientes objetivos específicos: $\diamond \quad$ Analizar la composición de las teorías de motivación de contenido: Maslow, Alderfer, McClelland y Herzberg.

$\diamond \quad$ Analizar la significancia estadística de las configuraciones (teorías de motivación), a través de los análisis de unidimensionalidad, validez y confiabilidad.

$\diamond \quad$ Analizar el peso relativo que las diferentes configuraciones (teorías de motivación) asignan a las necesidades.

\subsection{DISEÑO DE LA INVESTIGACIÓN}

La presente investigación es de tipo concluyente descriptiva con corte transversal o seccional (Malhotra, 2004), por cuanto se busca describir las teorías de motivación de contenido con la información recogida por la Universidad Diego Portales en la "Primera Encuesta Nacional de Satisfacción Laboral y Calidad de Vida (2009)".

En dicha encuesta, se asume que la percepción sobre la satisfacción con la vida puede ser explicada por un conjunto de dimensiones: sociales, familiares, de salud, ocio y trabajo (entre otras). Sin embargo, se considera que el foco de la presente investigación es estudiar la motivación laboral, por lo tanto solo se consideraron aquellas preguntas relacionadas con la satisfacción en el trabajo.

\subsubsection{MARCO MUESTRAL}

El estudio realizado por la Universidad Diego Portales consideró como marco muestral el Censo 2002. El universo estuvo compuesto por los habitantes de Chile, hombres y mujeres, mayores de 15 años, de sectores urbanos y rurales. Se consideró una muestra de 1500 personas, las cuales tienen representación estadística a nivel: 1) nacional, 2) Santiago, 3) por sexo y 4) por grupo etario.

\subsubsection{SELECCIÓN DE LA MUESTRA}

En la presente investigación solo se consideraron aquellas personas que tienen como actividad principal ser "trabajador dependiente", ya que el foco se encuentra en el análisis de la motivación en el ámbito laboral. Es así como, la muestra considera un total de 517 personas. 


\subsubsection{DESCRIPCIÓN DE LA MUESTRA}

La muestra de 517 personas ha sido caracterizada de acuerdo a variables sociodemográficas y laborales. Estas personas tienen una edad promedio de 39,05 años y el 46,2\% de los casos viven en la región metropolitana. El 56,9\% de la muestra tiene estudios de enseñanza media (33\%) o universitaria completa $(23,9 \%)$. Por otra parte, el $84,5 \%$ de la muestra tiene contrato indefinido (64,8\%) o definido (19,7\%), y el $52 \%$ de los casos se desempeña en una gran empresa.

\subsection{DETERMINACIÓN DE LAS DIMENSIONES}

Con el objetivo de obtener las tipologías de necesidades consideradas en los estudios de Maslow, Alderfer, McClelland y Herzberg, se procedió a realizar análisis factorial exploratorio con la metodología de Componentes Principales y Rotación Varimax (Hair et ál., 2005).

El número de factores a extraer fue forzado al número de necesidades consideradas en una determinada tipología (Malhotra, 2004). Se consideraron aquellos factores con valores de eigenvalue superior a 1 y de varianza explicada superior a un 60\% (Malhotra, 2004). Además, se consideraron aquellos indicadores (variables) que cargaban solamente sobre una dimensión (factor). Estas variables deben tener una varianza común con el resto de variables como mínimo de 0,4 (Hair et ál., 1998) y cargar sobre el componente con un valor mínimo de 0,5 (Larwood et ál., 1995).

\subsection{ANÁLISIS DE CONFIABILIDAD}

Una vez obtenidas las distintas tipologías de necesidades, se procedió a analizar la validez y confiabilidad de estas medidas. La confiabilidad de una medida analiza si esta se halla libre de errores aleatorios y en consecuencia, proporciona resultados estables y consistentes (Sánchez y Sarabia, 1999).

Para valorar la confiabilidad de una dimensión compuesta por tres o más variables se calcula el coeficiente Alpha de Cronbach. El valor del Alfa de Cronbach mínimo aconsejable para un análisis confirmatorio es de 0,7 ; cuando el análisis es de tipo exploratorio, el mínimo recomendado es de 0,6 . Por otra parte, para determinar la confiabilidad de dimensiones compuestas por dos variables se aplica el análisis de correlación; los valores obtenidos para las correlaciones deben ser significativos al 99\% o 95\% de confianza (Hair et ál., 1998).

\subsection{ANÁLISIS DE VALIDEZ}

La confiabilidad es una condición necesaria, aunque no suficiente, para la validez de dicha medida. La validez de una medida analiza si el instrumento utilizado mide lo que el investigador pretende medir; es decir, estudia el error sistemático: a menor error sistemático, mayor validez de la medida (Capelleras y Veciana, 2001).

La validez tiene tres aspectos: de contenido, discriminante y convergente (Nunnally, 1978). Considerando la naturaleza de la presente investigación es que se analizan los tres tipos de validez. La validez de contenido se consiguió al construir la escala con ítems considerados en estudios previos y con la opinión de expertos en el área (Deng y Dart, 1994). Por otra parte, la validez discriminante consideró la realización de Correlaciones de Pearson entre los componentes, por cuanto se busca que estas sean no significativas (García y Ruiz, 2007). Finalmente, la validez convergente se realizó a través del análisis de correlación entre la escala de medida y la satisfacción con la vida, por cuanto se busca que haya correlación entre dos variables distintas del mismo fenómeno (Capelleras y Veciana, 2001).

\subsection{ANÁLISIS DE UNIDIMENSIONALIDAD}

Es necesario verificar que cada una de las escalas (dimensiones) presenta suficientes grados de unidimensionalidad, lo cual implica que miden un único constructo. Con el objeto de identificar aquellos indicadores que menos están adheridos a un determinado factor, se aplican análisis factoriales de Componentes Principales (análisis factorial exploratorio) con Rotación Varimax (Hair et ál., 2005).

Cada una de las subescalas presenta un buen grado de unidimensionalidad cuando los ítems presentan cargas factoriales superiores a 0,4 (Larwood et ál., 1995). Además, los ítems 
deben cargar sobre un único factor y se deben presentar adecuados niveles de varianza explicada, KMO y test de Esfericidad de Bartlett (Hair et ál., 2005). Cuando uno o más indicadores cargan en más de un factor, se deben ir eliminando hasta conseguir la unidimensionalidad.

\section{DETERMINACIÓN DE LAS TEORÍAS DE MOTIVACIÓN}

Los análisis de componentes principales llevados a cabo son apropiados (con un nivel de confianza del 95\%), por cuanto en cada una de las teorías de motivación y el test de adecuación de la muestra (KMO) cumple con la condición de ser mayor o igual a 0,5. Además, en cada uno de los casos se cumple con que la probabilidad asociada al test de Bartlett ( $\mathrm{p}$-value) es menor al nivel de significancia de 0,05. Las dimensiones (en su conjunto) de cada una de estas teorías presentan valores de varianza explicadas (varianza acumulada) superiores a un $60 \%$ (Malhotra, 2004) (ver tabla 1).

TABLA 1

INDICADORES DE SIGNIFICANCIA DE LOS ANÁLISIS FACTORIALES

\begin{tabular}{cccc}
\hline $\begin{array}{c}\text { TEORÍA } \\
\text { DE MOTIVACIÓN }\end{array}$ & $\begin{array}{c}\text { MEDIDA DE ADECUACIÓN } \\
\text { DE LA MUESTRA (KMO) }\end{array}$ & $\begin{array}{c}\text { TEST DE ESFERICIDAD } \\
\text { DE BARTLETT }\end{array}$ & $\begin{array}{c}\text { VARIANZA ACUMULADA } \\
(\%)\end{array}$ \\
\hline Maslow & 0,783 & 0,000 & 73,006 \\
Alderfer & 0,579 & 0,000 & 67,758 \\
McClelland & 0,712 & 0,000 & 75,700 \\
Herzberg & 0,935 & 0,000 & 69,919 \\
\hline
\end{tabular}

Fuente: Elaboración propia.

Aunado a esto, en las tablas 2, 3, 4 y 5 se aprecia que el conjunto de variables consideradas en cada una de las teorías de la motivación, cumplen con el requisito de tener varianza común con el componente (comunalidad de extracción) como mínimo de 0,5 (Hair et ál., 1998). Asimismo, en cada una de las teorías analizadas, la carga de cada variable con el componente (carga factorial) cumple con el mínimo exigido de 0,4 (Larwood et ál., 1995).

\subsection{TEORÍA DE MASLOW}

Los 13 ítems fueron agrupados en 5 dimensiones que reflejan cada una de las necesidades consideradas en la Teoría de Maslow. El factor 1 corresponde a la "necesidad de autoestima”, por cuanto está compuesto por aspectos del trabajo que hacen a las personas sentirse bien consigo mismas. El factor 2 corresponde a la "necesidad de autorrealización", por cuanto está compuesto de aspectos laborales donde la persona ha podido realizar el propio potencial. El factor 3 corresponde a la "necesidad de seguridad", ya que trata sobre la estabilidad económica $y$ de sobrevivencia. El factor 4 corresponde a la "necesidad social", ya que trata sobre las relaciones que las personas establecen con los demás. Por último, el factor 5 corresponde a la "necesidad fisiológica", por cuanto hace referencia al estado de salud de las personas (ver tabla 2). 
TABLA 2

ANÁLISIS FACTORIAL DE LA TEORÍA DE MASLOW

\begin{tabular}{|c|c|c|c|c|c|c|}
\hline DIMENSIÓN & VARIABLES & $\begin{array}{c}\text { CARGAS } \\
\text { FACTORIALES }\end{array}$ & $\begin{array}{l}\text { COMUNALIDAD } \\
\text { DE } \\
\text { EXTRACCIÓN }\end{array}$ & EIGENVALUES & $\begin{array}{c}\text { VARIANZA } \\
\text { EXPLICADA } \\
(\%)\end{array}$ & $\begin{array}{c}\text { VARIANZA } \\
\text { ACUMULADA } \\
(\%)\end{array}$ \\
\hline \multirow[t]{4}{*}{$\begin{array}{l}\text { Necesidad } \\
\text { de } \\
\text { Autoestima }\end{array}$} & $\begin{array}{l}\text { El grado en que } \\
\text { puede desarrollarse } \\
\text { o crecer } \\
\text { personalmente en } \\
\text { su trabajo. }\end{array}$ & 0,836 & 0,747 & 2,651 & 20,396 & 20,396 \\
\hline & $\begin{array}{l}\text { El grado en que su } \\
\text { puesto de trabajo } \\
\text { le permite lograr } \\
\text { sus aspiraciones y } \\
\text { ambiciones. }\end{array}$ & 0,807 & 0,733 & & & \\
\hline & $\begin{array}{l}\text { Su impresión sobre } \\
\text { la forma en que se } \\
\text { le valora a usted y } \\
\text { los esfuerzos que } \\
\text { hace. }\end{array}$ & 0,784 & 0,649 & & & \\
\hline & $\begin{array}{l}\text { El grado en que } \\
\text { su trabajo le exige } \\
\text { hacer un esfuerzo } \\
\text { máximo. }\end{array}$ & 0,750 & 0,626 & & & \\
\hline \multirow{4}{*}{$\begin{array}{l}2 \\
\text { Necesidad } \\
\text { de } \\
\text { Autorrea- } \\
\text { lización }\end{array}$} & $\begin{array}{l}\text { Hasta ahora, he } \\
\text { conseguido las }\end{array}$ & 0,802 & 0,693 & 1,911 & 14,699 & 35,095 \\
\hline & $\begin{array}{l}\text { cosas que para mí } \\
\text { son importantes en } \\
\text { la vida. }\end{array}$ & & & & & \\
\hline & $\begin{array}{l}\text { Me siento orgulloso } \\
\text { de lo que he logrado } \\
\text { hasta ahora. }\end{array}$ & 0,745 & 0,657 & & & \\
\hline & $\begin{array}{l}\text { Me considero una } \\
\text { persona realizada. }\end{array}$ & 0,626 & 0,631 & & & \\
\hline $\begin{array}{l}3 \\
\text { Necesidad }\end{array}$ & $\begin{array}{l}\text { Estoy satisfecho(a) } \\
\text { con mi situación } \\
\text { económica. }\end{array}$ & 0,865 & 0,832 & 1,878 & 14,448 & 49,542 \\
\hline Seguridad & $\begin{array}{l}\text { Estoy satisfecho(a) } \\
\text { con lo que puedo } \\
\text { comprar. }\end{array}$ & 0,861 & 0,843 & & & \\
\hline \multirow[t]{2}{*}{$\begin{array}{l}4 \\
\text { Necesidad } \\
\text { Social }\end{array}$} & $\begin{array}{l}\text { Estoy satisfecho(a) } \\
\text { con los amigos que } \\
\text { tengo. }\end{array}$ & 0,902 & 0,826 & 1,681 & 12,930 & 62,472 \\
\hline & $\begin{array}{l}\text { Estoy satisfecho(a) } \\
\text { con mi vida social. }\end{array}$ & 0,853 & 0,810 & & & \\
\hline \multirow{2}{*}{$\begin{array}{l}5 \\
\text { Necesidades } \\
\text { Fisiológicas }\end{array}$} & $\begin{array}{l}\text { Tengo un buen } \\
\text { estado físico. }\end{array}$ & 0,854 & 0,783 & 1,369 & 10,534 & 73,006 \\
\hline & $\begin{array}{l}\text { Mi salud es } \\
\text { excelente. }\end{array}$ & 0,740 & 0,661 & & & \\
\hline
\end{tabular}

Fuente: Elaboración propia. 
Además, siguiendo la metodología de análisis factorial (Malhotra, 2004), es posible argumentar que para los trabajadores chilenos la necesidad de mayor importancia es la de "autoestima"; las siguientes necesidades en orden de importancia son las de autorrealización, seguridad, social y fisiológicas.

\subsection{TEORÍA DE ALDERFER}

Los 6 ítems fueron agrupados en 3 dimensiones que reflejan cada una de las necesidades consideradas en la Teoría de Aldefer. El factor 1 corresponde a la "necesidad de relación", ya que hace referencia al establecimiento de relaciones interpersonales. El factor 2 corresponde a la "necesidad de crecimiento", pues hace referencia a la necesidad de un continuo desarrollo y crecimiento personal. Por último, el factor 3 corresponde a la "necesidad de existencia", haciendo referencia a la necesidad de bienestar físico, ya sea a través de un adecuado estado de salud o de un salario que le permita satisfacer las necesidades básicas (ver tabla 3 ).

TABLA 3

ANÁLISIS FACTORIAL DE LA TEORÍA DE ALDERFER

\begin{tabular}{|c|c|c|c|c|c|c|}
\hline DIMENSIÓN & VARIABLES & $\begin{array}{c}\text { CARGAS } \\
\text { FACTORIALES }\end{array}$ & $\begin{array}{l}\text { COMUNALIDAD } \\
\text { DE EXTRACCIÓN }\end{array}$ & EIGENVALUES & $\begin{array}{c}\text { VARIANZA } \\
\text { EXPLICADA } \\
(\%)\end{array}$ & $\begin{array}{c}\text { VARIANZA } \\
\text { ACUMULADA } \\
(\%)\end{array}$ \\
\hline \multirow{2}{*}{$\begin{array}{l}1 \\
\text { Necesidad } \\
\text { de } \\
\text { Relación }\end{array}$} & $\begin{array}{l}\text { Estoy satisfecho(a) } \\
\text { con los amigos que } \\
\text { tengo. }\end{array}$ & 0,907 & 0,832 & 1,629 & 27,153 & 27,153 \\
\hline & $\begin{array}{l}\text { Estoy satisfecho(a) } \\
\text { con mi vida social. }\end{array}$ & 0,879 & 0,813 & & & \\
\hline \multirow{2}{*}{$\begin{array}{l}2 \\
\text { Necesidad } \\
\text { de } \\
\text { Crecimiento }\end{array}$} & $\begin{array}{l}\text { Tengo confianza en } \\
\text { mí mismo. }\end{array}$ & 0,758 & 0,596 & 1,311 & 21,854 & 49,007 \\
\hline & $\begin{array}{l}\text { Hasta ahora he } \\
\text { conseguido las } \\
\text { cosas que para mí } \\
\text { son importantes en } \\
\text { la vida. }\end{array}$ & 0,756 & 0,596 & & & \\
\hline \multirow[t]{2}{*}{$\begin{array}{l}3 \\
\text { Necesidad } \\
\text { de } \\
\text { Existencia }\end{array}$} & $\begin{array}{l}\text { Me pongo } \\
\text { enfermo(a) más } \\
\text { fácilmente que } \\
\text { otras personas. }\end{array}$ & 0,875 & 0,780 & 1,125 & 18,751 & 67,758 \\
\hline & $\begin{array}{l}\text { Su nivel salarial } \\
\text { en relación con su } \\
\text { experiencia. }\end{array}$ & 0,572 & 0,449 & & & \\
\hline
\end{tabular}

Fuente: Elaboración propia.

Además, siguiendo la metodología de análisis factorial (Malhotra, 2004), es posible argumentar que para los trabajadores chilenos la necesidad de mayor importancia es la de "relación"; las siguientes necesidades en orden de importancia son las de crecimiento $y$ existencia.

\subsection{TEORÍA DE MCCLELLAND}

Los 8 ítems fueron agrupados en 3 dimensiones que reflejan cada una de las necesidades consideradas en la Teoría de McClelland. El factor 1 corresponde a la "necesidad de logro", enfocándose en los 
objetivos de desarrollo y crecimiento personal que las personas buscan satisfacer en sus puestos de trabajo. El factor 2 corresponde a la "necesidad de poder", ya que se centra en la búsqueda de seguridad económica y de comprar todo aquello que se desea. El factor 3 corresponde a la "necesidad de afiliación", por cuanto hace referencia al establecimiento de relaciones interpersonales $y$ a la vida social en general (ver tabla 4).

TABLA 4

ANÁLISIS FACTORIAL DE LA TEORÍA DE MCCLELLAND

\begin{tabular}{|c|c|c|c|c|c|c|}
\hline DIMENSIÓN & VARIABLES & $\begin{array}{c}\text { CARGAS } \\
\text { FACTORIALES }\end{array}$ & $\begin{array}{l}\text { COMUNALIDAD } \\
\text { DE EXTRACCIÓN }\end{array}$ & EIGENVALUES & $\begin{array}{c}\text { VARIANZA } \\
\text { EXPLICADA } \\
(\%)\end{array}$ & $\begin{array}{c}\text { VARIANZA } \\
\text { ACUMULADA } \\
(\%)\end{array}$ \\
\hline \multirow[t]{4}{*}{$\begin{array}{l}\text { Necesidad } \\
\text { de } \\
\text { Logro }\end{array}$} & $\begin{array}{l}\text { Su impresión sobre } \\
\text { la forma en que se } \\
\text { le valora a usted y } \\
\text { los esfuerzos que } \\
\text { hace. }\end{array}$ & 0,838 & 0,950 & 2,628 & 32,847 & 32,847 \\
\hline & $\begin{array}{l}\text { El grado en que } \\
\text { puede desarrollarse } \\
\text { o crecer } \\
\text { personalmente en } \\
\text { su trabajo. }\end{array}$ & 0,804 & 0,843 & & & \\
\hline & $\begin{array}{l}\text { El grado en que su } \\
\text { puesto de trabajo } \\
\text { le permite lograr } \\
\text { sus aspiraciones y } \\
\text { ambiciones. }\end{array}$ & 0,798 & 0,876 & & & \\
\hline & $\begin{array}{l}\text { El grado en que } \\
\text { su trabajo le exige } \\
\text { hacer un esfuerzo } \\
\text { máximo. }\end{array}$ & 0,756 & 0,995 & & & \\
\hline \multirow{2}{*}{$\begin{array}{l}2 \\
\text { Necesidad } \\
\text { de } \\
\text { Poder }\end{array}$} & $\begin{array}{l}\text { Estoy satisfecho(a) } \\
\text { con lo que puedo } \\
\text { comprar. }\end{array}$ & 0,917 & 0,878 & 1,785 & 22,311 & 55,158 \\
\hline & $\begin{array}{l}\text { Estoy satisfecho(a) } \\
\text { con mi situación } \\
\text { económica. }\end{array}$ & 0,912 & 0,876 & & & \\
\hline \multirow{2}{*}{$\begin{array}{l}3 \\
\text { Necesidad } \\
\text { de } \\
\text { Afiliación }\end{array}$} & $\begin{array}{l}\text { Estoy satisfecho(a) } \\
\text { con los amigos que } \\
\text { tengo. }\end{array}$ & 0,907 & 0,859 & 1,643 & 20,541 & 75,700 \\
\hline & $\begin{array}{l}\text { Estoy satisfecho(a) } \\
\text { con mi vida social. }\end{array}$ & 0,877 & 0,844 & & & \\
\hline
\end{tabular}

Fuente: Elaboración propia.

Además, siguiendo la metodología de análisis factorial (Malhotra, 2004), es posible argumentar que para los trabajadores chilenos la necesidad de mayor importancia es la de "logro"; las siguientes necesidades en orden de importancia son las de poder y afiliación. 


\subsection{TEORÍA DE MOTIVACIÓN E HIGIENE}

Los 9 ítems fueron agrupados en 2 dimensiones que reflejan los factores considerados en la Teoría de Herzberg. El factor 1 corresponde a los "factores intrínsecos", ya que hacen referencia a características del entorno o ambiente laboral ajenas al puesto de trabajo y el factor 2 corresponde a los "factores extrínsecos", por cuanto hacen referencia a aspectos y características del puesto de trabajo (ver tabla 5).

TABLA 5

ANÁLISIS FACTORIAL DE LA TEORÍA DE HERZBERG

\begin{tabular}{|c|c|c|c|c|c|c|}
\hline DIMENSIÓN & VARIABLES & $\begin{array}{c}\text { CARGAS } \\
\text { FACTORIALES }\end{array}$ & $\begin{array}{l}\text { COMUNALIDAD } \\
\text { DE EXTRACCIÓN }\end{array}$ & EIGENVALUES & $\begin{array}{c}\text { VARIANZA } \\
\text { EXPLICADA } \\
(\%) \\
\end{array}$ & $\begin{array}{c}\text { VARIANZA } \\
\text { ACUMULADA } \\
(\%)\end{array}$ \\
\hline 1 & $\begin{array}{l}\text { El modo en que } \\
\text { se implantan }\end{array}$ & 0,821 & 0,736 & 3,748 & 41,646 & 41,646 \\
\hline \multirow{7}{*}{$\begin{array}{l}\text { Factores } \\
\text { Extrínsecos }\end{array}$} & los cambios o & & & & & \\
\hline & $\begin{array}{l}\text { innovaciones en su } \\
\text { organización. }\end{array}$ & & & & & \\
\hline & $\begin{array}{l}\text { El modo en que } \\
\text { se resuelven los } \\
\text { conflictos en su } \\
\text { organización. }\end{array}$ & 0,782 & 0,744 & & & \\
\hline & $\begin{array}{l}\text { La comunicación } \\
\text { y el modo en que } \\
\text { la información se } \\
\text { transmite en su } \\
\text { organización. }\end{array}$ & 0,740 & 0,538 & & & \\
\hline & $\begin{array}{l}\text { El estilo de } \\
\text { supervisión que } \\
\text { utilizan sus } \\
\text { superiores. }\end{array}$ & 0,715 & 0,733 & & & \\
\hline & $\begin{array}{l}\text { La estructura de la } \\
\text { organización. }\end{array}$ & 0,693 & 0,682 & & & \\
\hline & $\begin{array}{l}\text { El clima } \\
\text { psicológico que } \\
\text { predomina en su } \\
\text { organización. }\end{array}$ & 0,682 & 0,622 & & & \\
\hline \multirow{3}{*}{$\begin{array}{l}2 \\
\text { Factores } \\
\text { Intrínsecos }\end{array}$} & $\begin{array}{l}\text { El tipo de trabajo y } \\
\text { tareas que usted ha } \\
\text { de realizar. }\end{array}$ & 0,868 & 0,755 & 2,545 & 28,274 & 69,919 \\
\hline & $\begin{array}{l}\text { Su puesto de } \\
\text { trabajo actual en sí } \\
\text { mismo. }\end{array}$ & 0,802 & 0,826 & & & \\
\hline & $\begin{array}{l}\text { Su impresión sobre } \\
\text { la forma en que se } \\
\text { le valora a usted y } \\
\text { los esfuerzos que } \\
\text { hace. }\end{array}$ & 0,620 & 0,658 & & & \\
\hline
\end{tabular}

Fuente: Elaboración propia. 
Además, siguiendo la metodología de análisis factorial (Malhotra, 2004), es posible argumentar que para los trabajadores chilenos los factores de mayor importancia son los "extrínsecos" y en segundo lugar, aquellos relacionados con el propio trabajo "intrínsecos".

\section{ANÁLISIS DE VALIDEZ \\ 5.1. VALIDEZ DE CONTENIDO}

Para cada una de las tipologías de motivación analizadas, la validez de contenido se garantizó con la confirmación de las dimensiones (necesidades), a través del análisis de estudios previos e información secundaria (ver tabla 6). Además, el instrumento fue enviado a 3 expertos del área de comportamiento organizacional, quienes corroboraron que las preguntas seleccionadas miden alguna de las necesidades que constituyen las teorías de la motivación.

TABLA 6

ESCALAS UTILIZADAS EN LA CONSTRUCCIÓN DE LAS TEORÍAS DE MOTIVACIÓN

\begin{tabular}{ll}
\hline TEORÍAS DE MOTIVACIÓN & ESCALAS PREVIAS \\
\hline Maslow & Felce y Perry (1995); Gregg y Salisbury (2001); Rojas (2008); Swami y Chamorro (2009); \\
& Brown y Tierney (2009); Irmak y Kuruuzum (2009). \\
\hline \multirow{2}{*}{ Alderfer } & Felce y Perry (1995); Gregg y Salisbury (2001); Rojas (2008); Swami y Chamorro (2009); \\
& Brown y Tierney (2009); Irmak y Kuruuzum (2009). \\
\hline McClelland & Felce y Perry (1995); Gregg y Salisbury (2001); Rojas (2008); Brown y Tierney (2009); \\
& Irmak y Kuruuzum (2009). \\
\hline Herzberg & Gregg y Salisbury (2001); Rojas (2008); Liao (2009). \\
\hline
\end{tabular}

Fuente: Elaboración propia.

\subsection{VALIDEZ DISCRIMINANTE}

En lo que se refiere a la validez discriminante, se realizó un análisis de correlaciones entre las dimensiones obtenidas. Este análisis permitió demostrar que en cada una de las teorías de motivación analizadas, los coeficientes de correlación son nulos (ver tabla 7). 
TABLA 7

ANÁLISIS DE CORRELACIÓN DE LAS DIMENSIONES

\begin{tabular}{llcc}
\hline \multirow{2}{*}{ TEORÍAS DE MOTIVACIÓN } & COMBINACIONES & $\begin{array}{c}\text { CORRELACIÓN } \\
\text { DE PEARSON }\end{array}$ & SIG. (BILATERAL) \\
\hline Maslow & Fisiológicas-Seguridad & 0,000 & 1,000 \\
& Fisiológica-Social & 0,000 & 1,000 \\
& Fisiológica-Autoestima & 0,000 & 1,000 \\
& Fisiológica-Autorrealización & 0,000 & 1,000 \\
& Seguridad-Social & 0,000 & 1,000 \\
& Seguridad-Autoestima & 0,000 & 1,000 \\
& Seguridad-Autorrealización & 0,000 & 1,000 \\
& Social-Autoestima & 0,000 & 1,000 \\
& Social-Autorrealización & 0,000 & 1,000 \\
& Autoestima-Autorrealización & 0,000 & 1,000 \\
\hline Alderfer & Existencia-Relación & 0,000 & 1,000 \\
& Existencia-Crecimiento & 0,000 & 1,000 \\
& Relación-Crecimiento & 0,000 & 1,000 \\
\hline McClelland & Logro-Afiliación & 0,000 & 1,000 \\
& Logro-Poder & 0,000 & 1,000 \\
& Afiliación-Poder & 0,000 & 1,000 \\
\hline Herzberg & Extrínsecos-Intrínsecos & 0,000 & 1,000 \\
\hline
\end{tabular}

Fuente: Elaboración propia.

\subsection{VALIDEZ CONVERGENTE}

La validez convergente fue medida a través de la correlación entre la media de los valores de los ítems de la escala con la valoración de la satisfacción con la vida. Los resultados que se muestran en la tabla 8 , indican que en cada una de las teorías de motivación analizadas existe una correlación positiva y significativa entre ambas medidas. Dado esto, es posible señalar la existencia de validez convergente.

TABLA 8

ANÁLISIS DE CORRELACIÓN

ESCALAS DE MEDIDA Y SATISFACCIÓN CON LA VIDA

\begin{tabular}{|c|c|c|}
\hline $\begin{array}{c}\text { ESCALA } \\
\text { DE MEDIDA }\end{array}$ & CORRELACIÓN & $\begin{array}{l}\text { EN GENERAL, ¿QUÉ TAN SATISFECHO ESTÁ USTED } \\
\text { CON SU VIDA? }\end{array}$ \\
\hline \multirow[t]{2}{*}{ Maslow } & Correlación de Pearson & $0,445^{* *}$ \\
\hline & Sig. (bilateral) & 0,000 \\
\hline \multirow[t]{2}{*}{ Alderfer } & Correlación de Pearson & $0,416 * *$ \\
\hline & Sig. (bilateral) & 0,000 \\
\hline \multirow[t]{2}{*}{ McClelland } & Correlación de Pearson & $0,147 * *$ \\
\hline & Sig. (bilateral) & 0,001 \\
\hline \multirow[t]{2}{*}{ Herzberg } & Correlación de Pearson & $0,221 * *$ \\
\hline & Sig. (bilateral) & 0,000 \\
\hline
\end{tabular}

**La correlación es significativa al nivel 0,01 (bilateral).

Fuente: Elaboración propia. 
6. ANÁLISIS DE CONFIABILIDAD

Las escalas que miden cada una de las teorías de motivación son fiables, ya que presentan valores de Alfa de Cronbach superiores a 0,6 o valores de correlación de Pearson estadísticamente significativos con un nivel de confianza del 99\% (Hair et ál., 1998). Además, no fue necesario eliminar indicadores, por cuanto el valor del Alfa de Cronbach no se incrementa cuando se elimina alguno(s) de estos (ver tabla 9).

TABLA 9

ESTADÍSTICOS DE CONFIABILIDAD

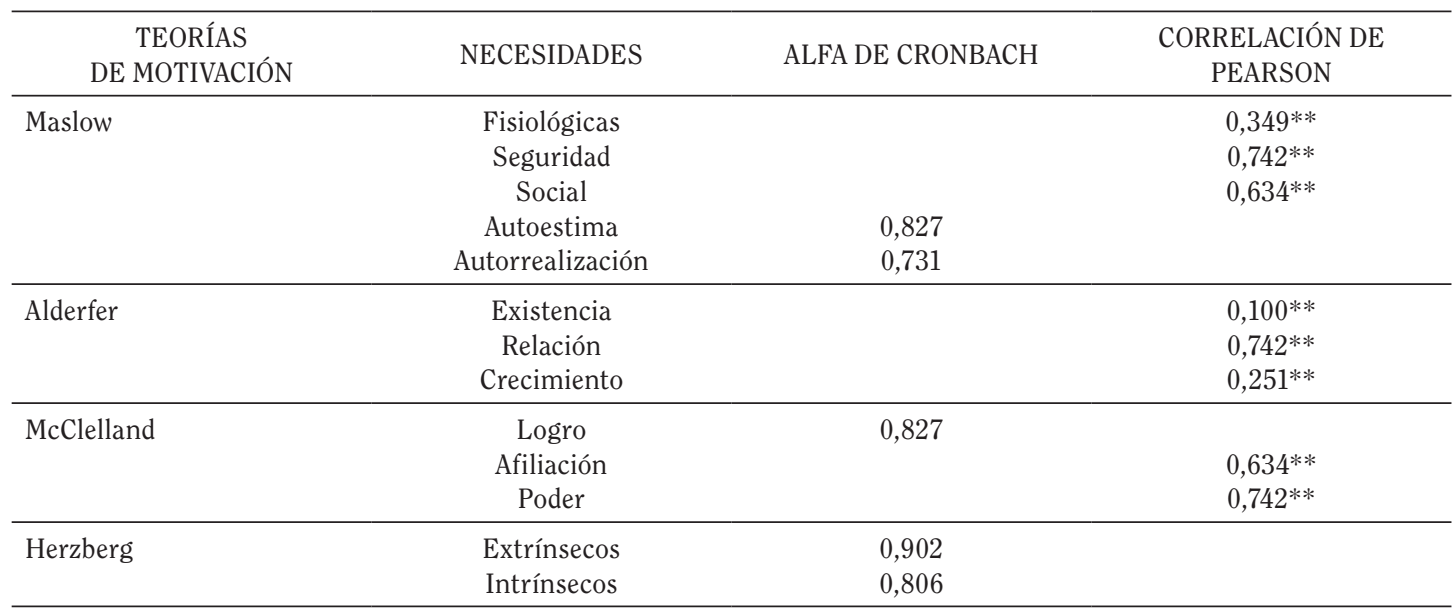

** La correlación es significativa al nivel 0,01 (bilateral).

Fuente: Elaboración propia.

\section{ANALISIS DE UNIDIMENSIONALIDAD}

Los resultados de los análisis factoriales realizados, permiten concluir que en cada una de las teorías de la motivación analizadas, las dimensiones (necesidades) presentan un buen grado de unidimensionalidad. Es así que en cada una de las teorías de motivación, los valores de KMO son superiores a 0,5 (Malhotra, 2004) y los valores de la varianza explicada son superiores a un 60\% (Hair et ál., 1998). Además, en cada una de las teorías de motivación, los indicadores presentan cargas factoriales superiores al mínimo exigido de 0,4 (Larwood et ál., 1995). 
TABLA 10

ESTADÍSTICOS DE UNIDIMENSIONALIDAD

\begin{tabular}{ccccc}
\hline $\begin{array}{c}\text { TEORÍAS } \\
\text { DE MOTIVACIÓN }\end{array}$ & DIMENSIONES & $\begin{array}{c}\text { TEST DE } \\
\text { ADECUACIÓN } \\
\text { DE LA MUESTRA } \\
(\text { KMO) }\end{array}$ & EIGENVALUES & $\begin{array}{c}\text { VARIANZA } \\
\text { EXPLICADA (\%) }\end{array}$ \\
\hline \multirow{4}{*}{ Maslow } & Fisiológicas & 0,500 & 1,349 & 67,452 \\
& Seguridad & 0,500 & 1,742 & 87,110 \\
& Social & 0,500 & 1,634 & 81,679 \\
& Autoestima & 0,779 & 2,634 & 65,857 \\
& Autorrealización & 0,680 & 1,954 & 65,146 \\
\hline \multirow{3}{*}{ Alderfer } & Existencia & 0,500 & 1,129 & 61,439 \\
& Relación & 0,500 & 1,634 & 81,679 \\
& Crecimiento & 0,500 & 1,251 & 62,550 \\
\hline \multirow{2}{*}{ McClelland } & Logro & 0,779 & 2,634 & 65,857 \\
& Afiliación & 0,500 & 1,634 & 81,679 \\
& Poder & 0,500 & 1,742 & 67,110 \\
\hline & Extrínsecos & 0,913 & 4,027 & 73,374 \\
\hline
\end{tabular}

Fuente: Elaboración propia.

\section{CONCLUSIONES}

En la presente investigación se configuraron y analizaron las principales teorías de motivación de contenido: Maslow, Alderfer, McClelland y Herzberg. Lo anterior es de importancia, ya que diversos autores han demostrado que trabajadores satisfechos presentan mayores niveles de desempeño.

Con este objetivo, se utilizó como base la información recogida por la Universidad Diego Portales en la "Primera Encuesta Nacional de Satisfacción Laboral y Calidad de Vida (2009)". Con una muestra de 517 trabajadores chilenos, se procedió a realizar análisis factorial de componentes principales, por cuanto esta metodología permitió la construcción de cada una de las teorías de motivación en estudio.

Los hallazgos de la presente investigación van en línea con lo señalado por la literatura, por cuanto se concluye que el mismo conjunto de ítems (variables) pueden ser agrupados en diferentes categorías (teorías de motivación). Los resultados son coherentes (no hay contradicciones entre las teorías de motivación) $y$ presentan adecuados niveles de unidimensionalidad, validez y confiabilidad.

A pesar de la validación estadística, se observan diferencias en la importancia que se atribuyen a las necesidades; estas diferencias no se observan entre las Teorías de Maslow y McClelland, por cuanto las dos necesidades de mayor importancia en la Teoría de Maslow (autoestima y autorrealización), coinciden con la necesidad que ocupa la primera posición en la Teoría de McClelland (logro). Pero, en el caso de la Teoría de Alderfer, la primera necesidad en importancia es la de relación, lo cual no coincide con los resultados de la Teoría de Maslow.

Sin embargo, esto no quita validez a los resultados obtenidos, ya que las diferencias se pueden explicar en el hecho de que la Teoría de Alderfer conceptualmente sólo representa una simplificación de la Teoría de Maslow. Dado esto, al reordenarse los ítems (variables), las dimensiones se ajustan en un orden distinto. Esto explica que la segunda necesidad en importancia en la Teoría de Alderfer es la de crecimiento, la cual representa las dos primeras posiciones en la Teoría de Maslow (autoestima y autorrealización). 
Aun cuando la presente investigación es un aporte en la literatura, tiene como limitación que la encuesta analizada no fue diseñada directamente para medir las teorías de motivación. Es así que otro tipo de instrumento podría permitir obtener conclusiones con niveles de interpretación más precisos.

No obstante, los resultados obtenidos podrían ser utilizados en investigaciones futuras. Por ejemplo, las teorías de la motivación obtenidas podrían ser comparadas y/o relacionadas con variables de desempeño laboral. Asimismo, en otras investigaciones se podrían utilizar los indicadores para construir instrumentos (escalas) que busquen replicar las teorías de motivación en contextos culturales o industriales específicos. Asimismo, los resultados podrían ser utilizados en la práctica profesional, ya sea en el diseño de planes de motivación o en la medición de los niveles de satisfacción laboral.

\section{REFERENCIAS}

\section{LIBROS}

Certo, S. C. Administración moderna. Octava edición. Colombia: Pearson Education, 2001.

Daft, L. Administración. Sexta edición. Thompson, 2004.

Ferrell, O. C. y Hirt, G. Introducción a los negocios en un mundo cambiante. Cuarta edición. McGraw-Hill, 2003.

Flores, C. Motivar a otros. Una experiencia fascinante. Venezuela: Fondo Editorial de la UPEL, 1996.

Hair, J.; Anderson, R.; Tatham, R. y Black, W. Multivariate data analysis. Fifth edition. Estados Unidos: Prentice Hall, 1998.

Hair, J.; Black, W.; Babin, B.; Anderson, R. y Tatham, R. Multivariate data analysis. Sixth edition. Reino Unido: Prentice-Hall International, 2005.

Hampton, R. Administración. Tercera edición. McGraw-Hill, 2000.

Herzberg, F.; Mausner, B. y Snyderman, B. The motivation to work. Estados Unidos: John Wiley, 1959.
Malhotra, N. Investigación de mercados. Cuarta edición. México: Pearson Educación, 2004.

McClelland, D. C. The achieving society. Princeton: Van Nostrand Reinhold, 1961.

Nunnally, J. Psychometric Theory. Second edition. Estados Unidos: McGraw-Hill, 1978.

Robbins, S. P. y Coulter, M. Administración. Octava edición. Pearson Prentice Hall, 2005.

Santrock, J. Psicología de la educación. México: McGraw-Hill, 2002.

Todt, E. La motivación. Barcelona: Herder, 1991.

\section{REVISTAS}

Alderfer, C. P. "An empirical test of a new theory of human needs". Organizational Behavior and Human Performance 4. 1969: 142-175.

Batista, A.; Gálvez, M. e Hinojosa, I. "Bosquejo histórico sobre las principales teorías de la motivación y su influencia en el proceso de enseñanza-aprendizaje". Revista Cubana de Medicina General Integral 26 (2). 2010: 376-386.

Bonillo, D. y Nieto, F. "La satisfacción laboral como elemento motivador del empleado". Trabajo 11. 2002: 189-200.

Brown, P. H. y Tierney, B. "Religion and subjective well-being among the elderly in China". The Journal of SocioEconomics 38. 2009: 310-319.

Brunstein, S.; Romero, R. y Albarrán, M. "Motivación hacia el estudio en el contexto universitario". Alternativas en Psicología 13. 2007: 19-25.

Deng, S. y Dart, J. "Measuring market orientation: a multi-factor, multiitem approach". Journal of Marketing Management 10 (8). 1994: 725-742.

Felce, D. y Perry, J. "Quality of life: its definition and measurement". Research in Developmental Disabilities 16 (1). 1995: 51-74.

García, P. M. y Ruiz, M. "Configuraciones organizativas en sectores dinámicos y hostiles: adecuación al contexto sectorial, 
coherencia interna y resultados". Cuadernos de Economía y Dirección de la Empresa 32. 2007: 111-148.

Gregg, P. M. y Salisbury, P. S. "Confirming and expanding the usefulness of the extended satisfaction with life scale". Social Indicators Research 54 (1). 2001: 1-16.

Irmak, S. y Kruuzum, A. "Turkish validity examination of the multidimensional students' life satisfaction scale". Social Indicators Research 92 (1). 2009: 13-23.

Larwood, L.; Falbe, C.; Kriger, M. y Miesing, P. "Structure and meaning of organizational vision". Academy of Management Journal 38 (3). 1995: 740-769.

Liao, P. "Parallels between objective indicators and subjective perceptions of quality of life: a study of metropolitan and county areas in Taiwan". Social Indicators Research 91 (1). 2009: 99-114.

Maslow, A. H. "A theory of human motivation". Psychological Review 50. 1943: 370-396.

Naranjo, M. "Motivación: perspectivas teóricas y algunas consideraciones de su importancia en el ámbito educativo". Revista Educación 33 (2). 2009: 153-170.

Rojas, M. "Experienced poverty and income poverty in Mexico: a subjective wellbeing approach". World Development 36 (6). 2008: 1078-1093.

Soler, A. y Chirolde, R. "Motivación y rendimiento docente en estudiantes bolivianos del nuevo programa de formación de médicos". Educación Médica Superior 24 (1). 2010: 42-51.

Soriano, M. "La motivación, pilar básico de todo tipo de esfuerzo". Revista de Relaciones Laborales 9. 2001: 163-184.

Swami, M. y Chamorro, T. "Psychometric evaluation of the Malay satisfaction with life scale". Social Indicators Research 92 (1). 2009: 25-33.

Wahba, M. A. y Bridwell, L. G. "Maslow reconsidered: a review of research on the need hierarchy theory". Organizational Behavior and Human Performance 15 (2). 1976: 212-240.

OTROS

Capelleras, J. L. y Veciana, J. M. "Calidad de servicio en la enseñanza universitaria: desarrollo y validación de una escala de medida". Documento de Trabajo 4. Departamento de Economía, Universidad Autónoma de Barcelona, 2001.

Instituto Nacional de Estadísticas (INE). "Censo 2002. Síntesis de resultados". Chile: INE, 2003. En: <http://www.ine.cl/cd2002/ sintesiscensal.pdf $>$.

Sánchez, M. y Sarabia, F. J. "Validez y fiabilidad de escalas". Metodología para la investigación en marketing y dirección de empresas. F. J. Sarabia (coord.). 1999: 363-392.

Fecha de ingreso: 16/11/2012 Fecha de aprobación: 23/01/2013 
